\title{
Motivating the Church-Turing Thesis in the Twenty-First Century
}

\author{
R. Gregory Taylor \\ Jersey City State College \\ Jersey City, New Jersey 07305 \\ taylor@jcs1.jcstate.edu
}

\section{ABSTRACT}

Theory of Computation students frequently

fail to appreciate the significance of the Church-Turing Thesis for one of two reasons. First, there is a tendency, on the part of students, to regard Church-Turing as tautologous and, consequently, devoid of important content. Second, there is a contrary impulse to view Church-Turing as unmotivated or even implausible. We describe our experience using simulation software in an effort to combat these two tendencies.

\subsection{Keywords}

Computability theory, Church-Turing Thesis, Turing machine, Markov algorithm, register machine, vector machine.

\section{INTRODUCTION}

We begin by recalling the usual formulation of the ChurchTuring Thesis:

Church-Turing Thesis. If (number-theoretic) function $f$ is effectively calculable, then $f$ is Turing-computablc. Equivalently, if function $f$ is not Turing-computable, then $f$ is not effectively calculable.

Properly understood, Church-Turing provides a concise summary of the classical Theory of Computation (see below). It constitutes a recurring theme in any good theory course. In addition, it provides an opportunity to relate Computer Science to the rest of the liberal arts curriculum (mathematics, the philosophy of mind, cognitive science, and even anthropology).

\footnotetext{
Permission to make digital or hard copies of all or part of this work for personal or classroom use is granted without fee provided that copies are not made or distributed for profit or commercial advantage and that copies bear this notice and the full citation on the first page. To copy otherwise, to republish, to post on servers or to redistribute to lists, requires prior specific permission and/or a fee.

ITiCSE '98 Dublin, Ireland

(C) 1998 ACM 1-58113-000-7/98/0008 .. \$5.00
}

Finally, it is a landmark of twentieth-century intellectual life. If students leave a theory course with anything, they should leave with an appreciation of Church-Turing. We believe that, often enough, this is not what happens.

\section{MATHEMATICS AND PHILOSOPHY}

Typically, if one's goal is to introduce the Church-Turing Thesis within the classroom, one begins by reviewing the concept of an algorithm. It will be emphasised that the algorithm concept is inexact to the extent that the notions used to characterise it-"next step," "carrying out," "result," and so forth-are being left rather vague. Students are asked to view the algorithm concept as part of the philosophy of mathematics.

Next, the concept of an effectively calculable function or effectively computable function is introduced-any function $f$, partial or total, for which an algorithm exists. Due to the use of the algorithm concept in characterising an effectively calculable function, the latter concept is, of course, similarly philosophical. One stresses the importance of not confusing the philosophical concept of an effectively computable function with the mathematically rigorous concept of a Turing-computable function: they are two distinct concepts-one philosophical (but concerning mathematics), the other genuinely mathematical.

- The philosophical notion of an effectively calculable function is one that underlies the culture of mathematics. It is part of every mathematician's informal sense of the subject matter.

- There is nothing informal about the concept of a Turing-computable function: a Turing-computable function is one computed by a single-tape Turing machine, where the latter is a quintuple of sets and functions satisfying certain conditions.

This is not to say that the two concepts-one philosophical and one mathematical-are unrelated, however, and that is precisely what Church-Turing is all about. Our point is that the two terms "effectively calculable function" and "Turingcomputable function" are not synonymous.

\section{EMPIRICAL JUSTIFICATION}

By virtue of the fact that Turing machines were intended as an analysis of the concept of computation, we take Turing 
in 1936 to be claiming that (1) number-theoretic function $f$ is effectively calculable if and only if $f$ is Turingcomputable. Students are reminded that (1) is not itself a mathematical proposition, since it makes use of the inexact notion of effective calculability. Hence it would be misguided to try to prove (1). Rather, one seeks to provide justification for it. Of course, since (1) is a biconditional, it has two parts: (a) If $f$ is effectively calculable, then $f$ is Turing-computable (Church-Turing) and (b) If $f$ is Turingcomputable, then $f$ is effectively calculable.

As claims about computation, (1a) and (1b) have different status. First, the student is encouraged to see that proposition ( $1 \mathrm{~b}$ ) is obvious. After all, if there is a Turing machine $M$ that computes $f$, then evidently $f$ is associated with an algorithm, namely, the algorithm embodied in the state diagram of $M$. On the other hand, (la) is not obvious: might there not be some effectively calculable function $f$ whose definition is so convoluted that no Turing machine could model the algorithm involved in computing this particular $f$ ? If so, (1a) would be false. (The mere fact that this question even makes sense means that Church-Turing is not obviously true.) Once it has been established that Church-Turing is not obvious, are there reasons to think that it might be true? Of course, the answer is yes, and now it is time to review a series of equivalence results.

- Assume that the first mathematical model of an effectively calculable function considered is the Turing machine model. An unaccountably infinite class of number-theoretic functions is seen to be partitioned into those that are Turing-computable and those that are not.

- Perhaps the partial recursive functions are introduced next. In that case, it is shown that the class of partial recursive functions is identical to the class of Turingcomputable functions.

- If Markov algorithms are considered, it is shown that the class of Markov-computable functions is none other than the class of Turing-computable functions (see Examples 2 and 3 below).

- Analogous equivalence results relate Turing computability to register-machine computability, Post computability, $\lambda$-definability, and vector-machine computability.

It is expected that students will be impressed by the fact that such apparently dissimilar proposals regarding computability should turn out, in the end, to capture the very same class of functions.

What we call the Argument from the Convergence of Dissimilar Ideas is introduced so as to establish the bearing of these equivalence results on the question of the truth of the Church-Turing Thesis. First, the fact that, for over 60 years now, every attempt to characterise the concept of computability has netted the same class of functions suggests that this class of functions is a "natural" classone such that the mathematical properties of its members make it the natural outcome of diverse analyses. The next step in the argument is to claim that this natural class can be none other than the class of effectively calculable functions. The empirical reasoning behind this second step is the following. We ask the question, If the class of Turingcomputable functions were, in fact, a proper subset of the class of effectively calculable functions, might we not expect that one or more of the alternative characterisations of computability would turn out to encompass functions that are not Turing-computable? But the plain fact is that, after many years of alternative models, none has produced a single function that does not demonstrably fall under Turing's model. This probably means that no such function exists.

\section{DECLINE OF THE COMPUTABILITY CONCEPT}

Some mention of arguments opposed to Church-Turing is always in order. But that is not our present focus. Rather, we wish to consider two obstacles to the instructor's instilling a proper apprcciation of Church-Turing and its importance for the Theory of Computation.

Although no student ever objects to either (1a) or (1b), we are convinced that many students do not understand either of them. And this is for one of two reasons. First, most students have no idea whatsoever of our intuitive concept of an effectively calculable function. Similarly, talk of our pretheoretic concept of computability is unlikely to have the intended effect. There is nothing unusual in this. It takes a long time to acquire a stance that is sufficiently objectiveor sufficiently philosophical-to enable a student to distinguish analysandum from analysans. More likely than not, having just acquired the notion of a Turing-computable function, the student conflates that technical notion (analysans) with the philosophical concept of a computable function (analysandum). ${ }^{1}$ In any case, no student ever questions Church-Turing, and we suspect that this is because, from the student's perspective, the two terms involved are synonymous anyway. One way to discourage this misconception is to address it directly by emphasising the distinction between an intuitive notion and the variety of technical concepts that have been introduced in order to characterise it. Since not much class time can be devoted to this issue, however, such direct efforts have, in the past, had but limited success.

\footnotetext{
${ }^{1}$ This tendency will be apparent to the extent that students use the terms "computable" and "Turing-computable" interchangeably without citing the Church-Turing Thesis. The situation becomes truly hopeless if the students' textbook appears to be doing the same thing.
} 
As we approach the twenty-first century, there is a second reason why our students fail to appreciate the remarkable claim embodied in the Church-Turing Thesis: namely, our students may well lack any robust, pretechnological concept of computation. Increasingly, computation is held to be the exclusive domain of calculators and digital computers. This may in turn mean that, from our students' point of view, the concept appearing in the antecedent of ( $1 \mathrm{a}$ ) belongs not to the philosophy of mathematics, as we have suggested, but, rather, to computer design theory. (In that case, the two concepts appearing in (1a) would both be viewed as technical in character.) Moreover, no mere slide-show of the history of computing (written records of manual reckoning, numerical tables used in calculating, historical computing devices, and so forth) is likely to reinstate what has been lost as the result of an omnipresent technology. ${ }^{2}$ Perversely, the solution that we propose involves introducing yet more technology.

Typically, instructors examine at most one alternative to Turing's model. It is then unsurprising that students have trouble distinguishing an intuitive notion of computability from Turing computability: Turing computability is the only (universal) notion of computability they have ever contemplated. Our remedy is to give students a rapid introduction to a number of analyses of computability, supported by software simulations of the models involved. In the past, it has been impossible to cover several models in a one-semester course. But this is no longer true, given the availability of software such as Deus ex machina designed by Nicolae Savoiu and available at http: //www.ics. uci.edu/ savoiu/dem.

Students eagerly investigate alternatives to the Turing machine model using this software. We review several examples below. (We highlight the name of any icon found in this software and in [3].)

\section{SOFTWARE SIMULATIONS}

\subsection{Example 1}

At icon LOGSPACE we present a three-tape, off-line Turing machine $M$ for converting the unary representation $I^{n+1}$ of natural number $n$ to the corresponding binary representation Moreover, $M$ visits only $\left\lceil\log _{2} n\right\rceil+3$

\footnotetext{
${ }^{2}$ Perhaps some readers will feel that we are overplaying this. But our experience has shown that at least some students-perhaps still a minority - have become dependent upon hand calculators even for very rudimentary computational tasks, e.g., integer division by 2 . What does this portend for the viability of a widespread, transcendent concept of effective calculability? Even those who would deny it any decisive impact will surely grant that this recent historical tendency can hardly strengthen our students' intuitive understanding of the nature of computation. Most likely, that understanding is thereby weakened. or so it seems to us.
}

squares on its middle tape. Accordingly, this busy little machine is seen to compute in logarithmic space.

\subsection{Example 2}

The four-tape Turing machine $M$ at icon Example 4.5.1 implements the Markov algorithm schema whose seven productions are displayed in Figure 1. Moreover, $M$ is the result of applying a completely general construction. It is thereby shown that any Markov-computable function is Turing computable.

\subsection{Example 3}

It is possible to describe a construction showing that the computational behaviour of any Turing machine can

$I^{*} 1 \rightarrow *$
$* \rightarrow \varepsilon$
$@ 111 \rightarrow @$
$@ 11 \rightarrow .111$
$@ 1 \rightarrow .11$
$@ \rightarrow .1$
$\varepsilon \rightarrow @$
Figure 1
be simulated by some Markov algorithm. Applied to a deterministic Turing machine $M$ computing $k$-ary function $f$, said construction yields a Markov algorithm schema $S$ that computes $f$. As an illustration, we apply the construction to a certain 13-state Turing machine $M$ computing unary function $f(n)=2 n$ (see Example 1.5.3 in the Turing folder). The result is the algorithm schema $S$ found at icon Example 4.5.2 within the Markov folder (see Figure 2). Since $M$ computes $f(n)=2 n$, so does $S$.

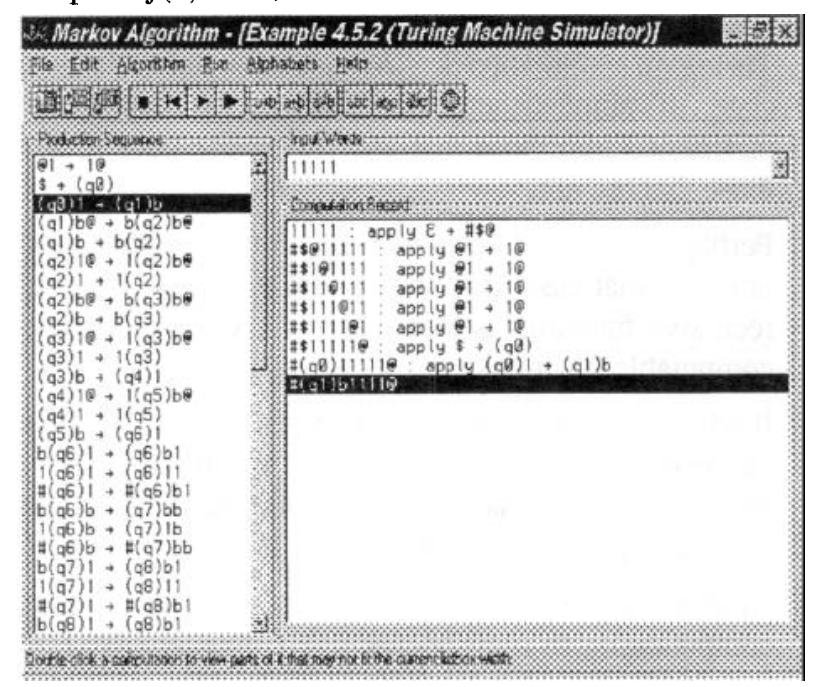

Figure 2

\subsection{Example 4}

In Figure 3 we present a snapshot of a register machine, in the sense of [2], that computes Ackermann's function. (Recall that the theoretical importance of Ackermann's function resides in the fact that it is a binary recursive function that is not primitive recursive.) 


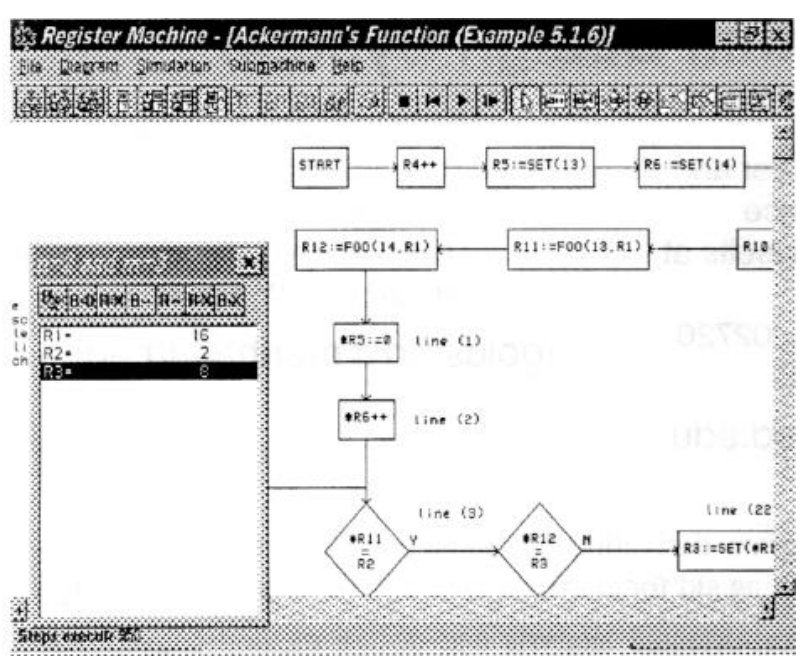

Figure 3

\subsection{Example 5}

The Vector Machine folder implements a theoretical model of parallel computation found in [1]. Figure 4 presents the flowchart of a vector machine that computes multiplication function $f(n, m)=n \cdot m$ (see Multiplication Machine).

It is our experience that, having been presented with several diverse technical analyses, the student is less likely to identify the intuitive notion of computability outright with any one of them. After all, to do so now would mean making an arbitrary choice. Consequently, the ChurchTuring Thesis is less likely to be seen as devoid of content, or such is our hope.

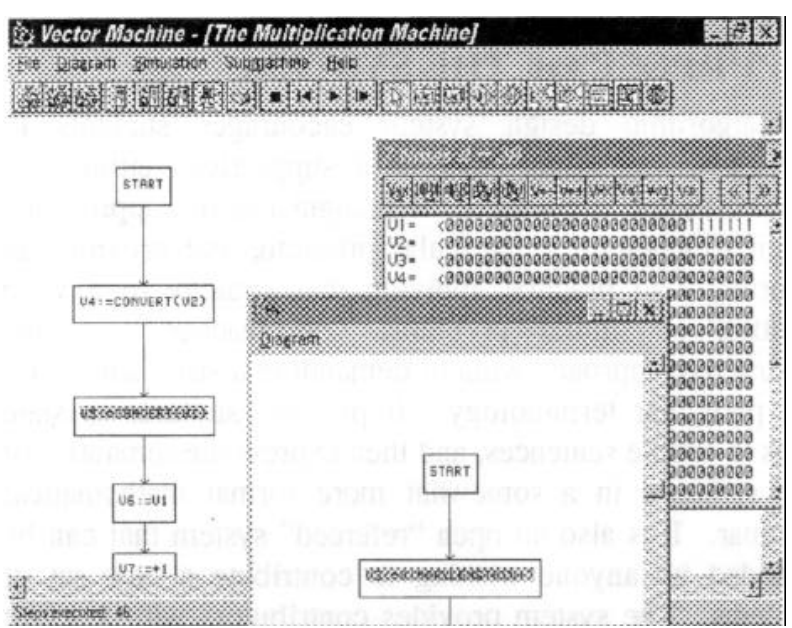

Figure 4
Finally, for some students, there is a second problem with respect to Church-Turing. Namely, once the student is convinced that Church-Turing really says something, there is the question of its truth. The plausibility of ChurchTuring is illustrated, most convincingly, by our last example.

\subsection{Example 6}

We present a five-tape Turing machine (C Interpreter) that compiles and runs programs written in a small (but growing) subset of $\mathrm{C}$, as described in the software documentation (see Figure 5).
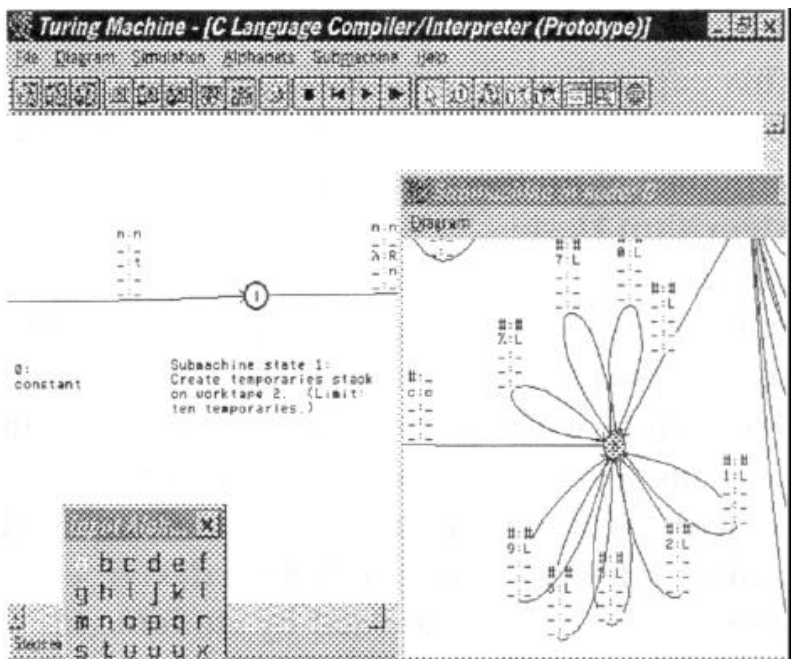

Figure 5

\section{ACKNOWLEDGMENTS}

We wish to thank Nicolae Savoiu for developing the software in which our examples have been created and Jane Stanton for editorial assistance.

\section{REFERENCES}

[1] Pratt, Vaughn R., and Stockmeyer, Larry J. A Characterisation of the Power of Vector Machines. Journal of Computer and System Sciences 12 (1976) 198-221.

[2] Shepherdson, J. C., and Sturgis, H. E. Computability of Recursive Functions. Journal of the Association of Computing Machinery 10 (1963) 217-255.

[3] Taylor, R. Gregory. Models of Computation and Formal Languages. Oxford University Press, New York, 1998. 